\title{
Caracterización de la evolución en el concepto de contabilidad de 1990 a 2010
}

doi:10.11144/Javeriana.cc15-37.cecc

\section{Maria Victoria Uribe-Bohórquez}

Administradora de Empresas y Contadora Pública de la

Pontificia Universidad Javeriana. Coordinadora del área de

contabilidad gerencial.

Correo electrónico: uribem@javeriana.edu.co 
Resumen La caracterización de la evolución en el concepto de contabilidad de 1990 a 2010 logra aportar referentes novedosos en el desarrollo académico e investigativo. Así mismo, el estudio del rol y alcance de esta disciplina en el entorno empresarial actual permite plantear su contribución a las pyme como componente fundamental de la reciente historia de la contabilidad.

Palabras clave autor: contabilidad; pyme; evolución; concepto; enfoque

Palabras claves descriptor: contabilidad-19902010; pequeña y mediana empresa

Códigos JEL: M 40

\section{Characterization of the Evolution in the Concept of Accounting from 1900 to 2010}

\begin{abstract}
The characterization of the evolution in the concept of accounting from 1990 to 2010 contributes innovative elements in academic and research development. Likewise, studying the role and scope of this discipline in the current corporate environment allows planning its contribution to Small and Medium-Sized Companies, as an essential component of the recent history of accounting.
\end{abstract}

Key words author: accounting; pyme (small and medium-sized companies); evolution; concept; approach

Key words plus: accounting- 1990-2010; small and medium-sized companies

\section{Caracterização da evolução no conceito de Contabilidade de 1990 até 2010}

Resumo A caracterização da evolução no conceito de Contabilidade de 1990 até 2010 consegui trazer referentes novos no desenvolvimento acadêmico e pesquisador. Assim mesmo, o estudo do papel e alcance desta disciplina no entorno empresarial atual permite levantar sua contribuição às PMEs como componente fundamental da recente história da Contabilidade.

Palavras-chave autor: contabilidade; PMEs; evolução; conceito; enfoque

Palavras-chave descritor: contabilidade- 19902010; pequenas e médias empresas

\section{Introducción}

El presente documento pretende revelar las características de las definiciones y/o enfoques de la contabilidad de 1990 a 2010, y tipificarlas de acuerdo al rol y alcance que se les da en las pyme. Para esto se tuvo en cuenta un número importante de autores que se espera sea representativo de todas las corrientes que se generaron en esta época, lo cual se realizó a partir de revisiones en publicaciones seriadas, libros y documentos en internet.

Como este se considera un ejercicio de reunión y revelación asume todas las concepciones de manera igualitaria, incluso en algunos casos de forma complementaria y asume como superada la discusión de si la contabilidad se considera ciencia, técnica, tecnociencia o arte.

El horizonte de tiempo se elige pensando en las consecuencias de la apertura económica 
que durante los años noventa tuvo sus inicios en Colombia y ya mostraba su impacto en otros países de Latinoamérica, lo cual generó cambios en el modelo económico que sin duda tuvieron repercusiones importantes en las empresas y-por ende, se intuiría- en la contabilidad.

A partir de lo ya enunciado, se espera generar reflexiones en dos escenarios, el primero en las pyme en cuanto a su concepción actual y potencial de la contabilidad y en la academia, al abrir puertas al análisis de enfoques históricos y tradicionales frente a los contemporáneos, la contraposición de conceptos y el análisis desde la ética, entre otros temas.

\section{Contabilidad en las pyme}

En aras de reunir y tipificar las definiciones de contabilidad, fue posible encontrar algunas agrupaciones representativas; no obstante, estas atendían a exigencias y visiones variadas, algunas de ellas coincidentes y otras no. Por tanto, estas se asociarán teniendo como criterio el rol que juega la contabilidad dentro de las pyme. Vale la pena aclarar que si bien se busca catalogarlas dentro de alguna visión, muchas de ellas tienen rasgos diversos y por ellos se pudieran incluir en más de un enfoque.

Así, en el primer grupo "Enfoque tradicional" se asocian aquellas definiciones que asumen criterios históricos, tradicionales o incluso instrumentales como aspecto esencial en el uso que las pyme le podrían dar a la contabilidad; por ejemplo, entenderla como la mera ejecución de una actividad profesional, una función de registro, el desarrollo de un proceso, un ejer- cicio puramente jurídico y de cumplimiento, así como la tarea de medir el patrimonio. Aunque esta última clasificación puede verse como un asunto "tradicional" o como uno estratégico cuando se piensa en la gestión del patrimonio (siguiente enfoque).

En el segundo grupo "Enfoque de utilidad" se ubican aquellas definiciones en que las pyme y las empresas en general reconocen en la contabilidad una fuente de información que ayuda a tomar decisiones adecuadas. Como su nombre lo indica, que sea útil en la gestión del patrimonio-riqueza y entiende a estas organizaciones en un entorno económico; en este último caso, algunas definiciones incluso trascienden a reconocer que también hay una dimensión social en esa actividad económica (tercer enfoque).

Por último, en el "Enfoque de reconocimiento del entorno" se vislumbra una disciplina con responsabilidad y de carácter social que atiende a un entorno y permite su desarrollo no solo en términos económicos, es decir, le da una orientación macro.

\section{Enfoque tradicional}

A pesar de que la elección del horizonte de tiempo está motivada por los cambios socioeconómicos que se han dado entre 1990 y 2010 , hay una corriente que concibe la contabilidad desde un enfoque primordialmente instrumental y que sigue preceptos históricos.

Algunos autores entienden la contabilidad desde el mero ejercicio, lo cual omite la esencia propia de la ciencia y se traslada al quehacer de quien lo ejecuta. 
Contabilidad se refiere a las actividades profesionales realizadas por contadores, como la preparación y la auditoría de estados financieros, el mantenimiento de registros financieros y asesoría en materia de impuestos y otros asuntos financieros (Aarthur School of Business).

Contabilidad se refiere a la accountancy, es decir, las actividades profesionales realizadas por contadores, como la preparación y la auditoría de estados financieros, el mantenimiento de registros financieros y la asesoría en materia de impuestos y otros asuntos financieros (Aarthur School of Business).

Otra corriente es aquella que vincula la contabilidad con un criterio legalista, si bien es innegable el reconocimiento que esta hace de las relaciones jurídicas, algunas definiciones sitúan la esencia de la contabilidad como el resultado de un requisito o el origen de reglas.

'La contabilidad es la ciencia que enseña las reglas que permiten registrar las operaciones efectuadas por una o varias personas (L. Batardon - francés)' (Chaves, Chyrikins, Dealecsandris, Fronti de García, Pahlen-Acuña \& Viegas, 1998).

La contabilidad ayuda a poner en práctica y a hacer cumplir los contratos que constituyen la empresa. La contabilidad desempeña cinco funciones para posibilitar el funcionamiento de la empresa.

1. Mide las contribuciones de cada agente al fondo común de recursos de la empresa.

2. Determina y liquida el derecho contractual de cada agente.
3. Informa a los agentes apropiados hasta qué punto los otros agentes han cumplido sus obligaciones contractuales y han recibido sus derechos.

4. Ayuda a mantener un mercado líquido para las participaciones contractuales y para los factores de producción ofrecidos por sus participantes, de manera que la rescisión o terminación de un agente no amenace la existencia de la empresa.

5. Como los contratos de varios agentes son periódicamente renegociados, provee una base de conocimiento común de información verificada a todos los participantes para facilitar la negociación y la formalización de los contratos. ...La contabilidad en sí misma es una parte del conjunto de los contratos los cuales esta ayuda a operar... (Sunder, 2007).

No obstante, autores como Jorge Tua-Pereda (2000) comprenden su carácter más allá del netamente jurídico, como se ve a continuación: “...La contabilidad ha pasado de ser un instrumento de carácter casi exclusivamente jurídico en defensa de su propietario, y con finalidad de registro, a convertirse en una disciplina científica, con aplicación preferente a la actividad económica - en la medida en que otras aplicaciones ajenas son todavía meros esbozosy con evidentes rasgos de carácter social, por su naturaleza como disciplina científica, por el tipo de información que suministra, y por su capacidad de servicio en cuanto a la satisfacción de las necesidades de sus usuarios en orden a facilitarles la toma de decisiones...”. 
Así mismo, otras versiones siguen corrientes tradicionales e instrumentales que vinculan la funcionalidad de la contabilidad al registro, partiendo principalmente del reconocimiento y atribuyendo la esencia a los libros.

La contabilidad es una técnica que se utiliza para el registro de las operaciones que afectan económicamente a una entidad y que produce sistemática y estructuradamente información financiera (Institute of Accounting Standards Board, IASB, 2009).

La contabilidad de una empresa o de un país es un registro numérico de todos los flujos (productos, costes, etc.) de un determinado período (Samuelson, Nordhaus \& Mandel, 1996).

'La contabilidad es la ciencia que coordina y dispone en libros adecuados las anotaciones de las operaciones efectuadas por una empresa mercantil, con el objeto de poder conocer la situación de dicha empresa, determinar los resultados obtenidos y explicar las causas que han producido estos resultados (Boter-Mauri, español)' (Chaves, Chyrikins, Dealecsandris, Fronti de García, Pahlen-Acuña \& Viegas, 1998).

Las siguientes concepciones van en el mismo sentido instrumental y ponen como eje el proceso que se lleva a cabo con las transacciones:

La contabilidad es el proceso de actividades que incluyen la identificación, medición y reconocimiento de las transacciones de la empresa e incluye la contabilidad financiera y gerencial (Aarthur School of Business).
La contabilidad es el proceso de identificar, registrar y comunicar los sucesos económicos de una organización en particular a los usuarios interesados (Kieso, Kimmel, \& Calvet-Pérez, 2008)

Es la técnica mediante la cual se puede identificar, medir, clasificar, registrar, interpretar, analizar, evaluar e informar las operaciones de un ente económico en forma completa y fidedigna con sujeción a los principios y normas generalmente aceptadas (Sánchez, 2003).

Contabilidad es el arte de registrar, clasificar y resumir de una manera significativa, y en términos monetarios, operaciones y hechos que tienen, por lo menos en parte, un carácter financiero, e interpretar los resultados de dichos hechos y operaciones (Chaves, Chyrikins, Dealecsandris, Fronti de García, Pahlen-Acuña \& Viegas, 1998).

Así mismo, hay autores que vinculan la contabilidad como ciencia al servicio del patrimonio, como se puede ver a continuación:

Es la ciencia que estudia el patrimonio, y las variaciones que en él se producen. El objeto de la contabilidad es la medición adecuada del patrimonio en sus diversos aspectos: Estático: valor del patrimonio en una fecha determinada.

Dinámico: variación del patrimonio a lo largo de un período de tiempo.

Cuantitativo: valor de los elementos integrantes del patrimonio.

Cualitativo: cuáles son los elementos que componen el patrimonio. 
Económico: inversiones realizadas por la empresa con los fondos obtenidos.

Financiero: dónde se han obtenido los fondos con los que se realizaron las inversiones (Rey-Pombo, 2006).

La contabilidad es una ciencia económica que estudia el patrimonio de las empresas en sus aspectos cualitativos y cuantitativos estáticos y dinámicos

La contabilidad determina:

Qué elementos forman el patrimonio de la empresa.

Qué valor se le da a cada uno.

Cuál es la situación de todos sus elementos en un momento determinado.

Cuál es la evolución de todos y cada uno de sus elementos en el tiempo (Delgado-González \& Ena-Ventura, 2006).

Rama de la ciencia empresarial que trata sobre la forma de registrar las variaciones que experimentan los patrimonios de las empresas, sociedades, comerciantes individuales, instituciones públicas o privadas, etc., así como de la cantidad y clase de las pérdidas o ganancias que estos tengan como producto de su actividad empresarial. Es, en definitiva, una forma de reflejar ordenadamente las variaciones patrimoniales con el objeto de llevar un control exacto de todas las operaciones, así como de los resultados que estas arrojan... (Martín-Amez, 2002).

La contabilidad es una ciencia económica, su objeto es el patrimonio, en sus aspectos estáticos y dinámicos, cualitativos y cuantitativos su fin hace referencia a la representación de dicho patrimonio, lo cual exige una captación y cuantificación previas (TascónFernández, 1995).

La contabilidad va a representar el patrimonio en su aspecto económico, cualitativa y cuantitativamente - es decir, los elementos que forman ese patrimonio debidamente descritos y valorados-al comenzar el ejercicio económico (Rivero-Romero, 1991).

Estas últimas definiciones nos hacen pensar en las decisiones que tienen que tomar los administradores y empresarios con su riqueza (patrimonio) y así la Contabilidad no solo se puede encuadrar en enfoques instrumentales sino que trasciende a uno que ayude a gestionarla, pensando en esto y en el uso que las pyme le puedan dar a la contabilidad, pasemos al "enfoque de utilidad".

\section{Enfoque de utilidad}

Una de las concepciones generalizadas y de mayor aceptación es la relacionada con la contabilidad como un sistema de información y, en consecuencia, como un sistema de control, como se evidencia con las siguientes definiciones:

Hoy por hoy, la contabilidad es entendida universalmente como una disciplina científica. Esta concepción de la contabilidad potencia su desarrollo como sistema de información y de control organizacional (Consejo Técnico de la Contaduría Pública, (TCP).

La contabilidad es la disciplina que sirve para proporcionar información útil en la 
toma de decisiones económicas (Instituto Mexicano de Contadores Públicos, IMCP). Contabilidad es el proceso de identificar, medir y comunicar información económica que permitan decisiones y juicios informados por los usuarios de la información" (Institute of Chartered Accountants in England and Wales, ICAEW).

Sistema que permite identificar, medir, procesar y comunicar información contable para tomar decisiones, emitir juicios y ejercer la función de control (Contaduría General de la Nación, 2009).

La contabilidad constituye un método de registro y un sistema de información y de control del fenómeno económico y financiero que tiene lugar en el proceso circulatorio y de creación de valores que constituye el soporte para la toma de decisiones en la unidad económica (Sánchez-Fernández de Valderrama, 2008).

Registro, clasificación y resumen de eventos económicos en una forma lógica con el objeto de proporcionar información financiera para la toma de decisiones (Arens, Elder \& Beasley, 2007).

La contabilidad es un proceso de información acumulativo de la empresa, cuyas funciones son: recolectar, identificar, medir, clasificar, codificar, acumular, registrar, emitir estados financieros, interpretar, analizar, evaluar e informar, y hacer el seguimiento al desarrollo de las operaciones de un ente económico, en forma clara, completa y fidedigna (FierroMartínez, 2007).

La contabilidad está centrada en la capacidad que tienen los profesionales que la ejercen de recopilar y ordenar datos para procesarlos y obtener información financiera, ambiental y social. Información, que deberá ser útil, comprensible y comparable, en múltiples ámbitos de toma de decisiones sociales y para múltiples usuarios que la toman como base cierta de actuación en medio de una realidad incierta y cambiante (Agudelo-Henao, 2006).

Es un sistema de información que permite recopilar, clasificar y registrar de una forma sistemática y estructural, las operaciones mercantiles realizadas por una empresa, con el fin de producir informes que, analizados e interpretados, permitan planear, controlar y tomar decisiones sobre la actividad de la empresa (Gudiño-Dávila \& Coral-Delgado, 2005).

La contabilidad constituye un sistema de información integrado a la empresa, cuyas funciones son identificar, medir, clasificar, registrar, interpretar, analizar, evaluar e informar las operaciones de un ente económico, en forma clara, completa y fidedigna (Sinisterra-Valencia, Polanco-Izquierdo \& Henao, 2005).

El objetivo de la contabilidad es generar y comunicar información útil para la oportuna toma de decisiones de los acreedores y accionistas de un negocio, así como de otros públicos interesados en la situación financiera de una organización (Guajardo-Cantú, 2004).

La contabilidad es un sistema de información que clasifica, registra, resume y presenta las transacciones de entidades económicas, en cuanto tales transacciones 
constituyen movimientos de dinero o bienes representables en dinero, con el fin de determinar sus resultados, interpretarlos y analizarlos, proyectar las finanzas de la empresa y controlar la ejecución de sus planes y la veracidad de las operaciones realizadas y de su registro.

Se dice que es un sistema de información porque recoge la información de todos los ámbitos de la empresa, la procesa y la distribuye adentro y fuera de la compañía, a todas las personas y entidades interesadas en ella (Carrillo de Rojas, 2004).

La contabilidad se puede definir como un sistema de información que permite describir y comprender la actividad económica de la empresa de manera resumida, para la toma de decisiones...

...Se puede sintetizar que el propósito fundamental de la contabilidad es proporcionar datos financieros que sirvan para medir la rentabilidad del negocio y la solvencia que tiene para cumplir sus compromisos (Escoto, 2004).

Sistema de información que mide las actividades de las empresas, procesa esa información en estados (informes) y comunica los resultados a los tomadores de decisiones (Horngren, Harrison \& Smith, 2003).

Es un sistema de información integrado a la empresa cuyas funciones son recolectar, clasificar, resumir, registrar e informar los hechos económicos realizados por una persona natural o jurídica, durante un período determinado. La contabilidad coadyuva a las funciones administrativas de planeación, control y toma de decisiones oportunas y acertadas.
También se dice que la contabilidad es el lenguaje de los negocios. A través de ella conocemos la situación socioeconómica general y el resultado de las operaciones sociales realizadas por un ente económico.

Hoy en día se define la contabilidad como un servicio integral de información de hechos cuantitativos pasados que sirve para la gestión administrativa y operativa de la organización (López-Ramírez, 2002).

El objetivo principal de la contabilidad es proporcionar información útil en la toma de decisiones...

...El producto final de la información contable es la decisión, ampliada en último término por el uso de la información contable...

...Algunas veces se hace referencia a ella como el lenguaje del negocio...

...La contabilidad 'une' a quienes toman decisiones con las actividades económicas y con los resultados de sus decisiones (Meigs, Williams, Haka \& Bettner, 2000).

La contabilidad es una disciplina o rama del conocimiento que permite controlar las operaciones del ente; también suministra datos útiles para todos los interesados en los informes que produce la contabilidad, y además facilita una larga lista de actividades vinculadas con el ente (Biondi, 1999).

La Contabilidad es un sistema de información, sujeto a una serie de normas que garanticen su objetividad. Su objeto es la realidad socioeconómica. Su fin es facilitar información, para la toma de decisiones y el control por parte de los usuarios de la misma (Tascón-Fernández, 1995). 
La Contabilidad, parte integrante del sistema de información de un ente, es la técnica de procesamiento de datos que permite obtener información sobre la composición y evolución del patrimonio de dicho ente, los bienes de propiedad de terceros en poder del mismo y ciertas contingencias. Dicha información debería ser de utilidad para facilitar las decisiones de los administradores del ente y de los terceros que interactúan o pueden llegar a interactuar con él, así como para permitir una eficaz vigilancia sobre los recursos y obligaciones del ente (FowlerNewton, 1992).

La contabilidad es una ciencia de naturaleza económica que tiene por objeto producir información para hacer posible el conocimiento pasado, presente y futuro de la realidad económica en términos cuantitativos a todos sus niveles organizativos, mediante la utilización de un método específico apoyado en bases suficientemente contrastadas, con el fin de facilitar la adopción de las decisiones financieras externas y las de planificación y control internas (Cañibano-Calvo, 1990). La contabilidad tiene varios aspectos. Primero, es un sistema de información en sí mismo. Es decir, emplea varias operaciones sistemáticas para generar información relevante. Dentro de las operaciones que esta abarca están (1) el registro de los datos económicos (recolección de datos), (2) mantenimiento de los datos almacenados (mantenimiento de datos), y (3) presentación de información cuantitativa en términos financieros (generación de información)... Segundo, la contabilidad es el 'lenguaje de los negocios': proporciona los medios por los cuales los asuntos clave de una firma de negocios se expresan y resumen. Finalmente, la contabilidad puede ser vista como la información financiera necesaria para el funcionamiento general de una entidad (como una firma de negocios) (Wilkinson, Cerullo, Raval \& Wong-On-Wing, 2000).

Como se puede ver con el gran número de definiciones, esta es la corriente en la que se han generado un mayor número de documentos y de conceptos, no obstante en algunos casos se parte de un proceso estático y deja al contador como una persona que se limita a una tarea operativa, incluso algunos piensan en la contabilidad como un procesamiento de datos; sin embargo, algunos autores han relacionado sus definiciones con ello.

La definición de contabilidad ha evolucionado a lo largo del tiempo. Tradicionalmente, la contabilidad se ha definido como el 'lenguaje de los negocios' por el hecho de ser, dentro de la organización, una actividad de servicio encargada de identificar, medir y comunicar la información económica que permite a los diferentes usuarios formular juicios y tomar decisiones. También se ha conocido como la 'historia de los negocios', pues la contabilidad registra cronológicamente lo que sucede en una empresa. Con los avances tecnológicos, la contabilidad ha presentado considerable aplicación en todo tipo de empresas, en las agencias del Estado y en las entidades sin ánimo de lucro, 
convirtiéndose en un poderoso instrumento de información.

...Independientemente que se trate de una técnica, un arte o una ciencia, la contabilidad constituye un sistema de información integrado a la empresa, cuyas funciones son identificar, medir, clasificar, registrar, interpretar, analizar, evaluar e informar las operaciones de un ente económico, en forma clara, completa y fidedigna.

Las actividades de clasificación y registro son de carácter rutinario y repetitivo, y no constituyen funciones finales de la contabilidad. El desarrollo y la sistematización de la contabilidad han liberado al contador de esta fase del proceso, lo que le permite dedicar más tiempo a labores de mayor importancia, como el análisis y la interpretación de la información (Sinisterra-Valencia, Polanco-Izquierdo \& Henao, 2001).

Las siguientes definiciones dependiendo del uso y el entendimiento que se les dé, pueden encuadrar tanto en el "Enfoque de utilidad" como en el "Enfoque de reconocimiento del entorno". Por ejemplo, algunos autores mencionan que la contabilidad permite un análisis y un conocimiento de la realidad económica pero algunas lo identifican en el marco de un sistema circulatorio superior que incluso reconoce una dimensión social. Veamos algunos casos:

...Nuestra posición es que la contabilidad es un conocimiento que permite la representación de la naturaleza económica y la representación del contenido financiero de la realidad (que resulta subsumida en la teoría económica), a la cual le aporta una metodología propia de captación, registro y revelación que permiten el análisis, la interpretación y la proyección

Ese conocimiento asume la caracterización de una tecnociencia social. Su campo es el reconocimiento, captación, sistematización, medición, registro y diseño normalizado de ciertas dimensiones de la realidad económica, a fin de generar información. Entre sus operaciones se incluyen el análisis, la interpretación y proyección, que permiten transformar la información en conocimiento aplicado al proceso decisorio previo a la acción. La contabilidad permite conocer para actuar y éste es su verdadero valor agregado. En la denominada Era de la información y en un marco globalizado, no puede prescindirse de este enfoque (Gil, 2010). La contabilidad posee naturaleza científica, quedando encuadrada dentro de las ciencias económicas.

$\mathrm{Su}$ objeto material es la realidad (socio) económica de las unidades económicas.

Su objeto formal es el conocimiento cualitativo y cuantitativo de dicha realidad.

Su fin genérico es determinar la situación de la unidad económica y su evolución en el tiempo con diferentes propósitos específicos, como facilitar la toma de decisiones. Para lograr su fin, utiliza métodos para la captación, medida, valoración, representación y comunicación de la información de los hechos, expresados normalmente en términos cuantitativos, aunque también facilita información de carácter cualitativo (DénizMayor, Bona-Sánchez, Pérez-Alemán \& Suárez-Falcón, 2008). 
Hoy en día, la contabilidad no se aprecia como una técnica estática, sino como una disciplina del conocimiento, dinámica, que dentro del contexto social mide, evalúa y proyecta el crecimiento y desarrollo económico de cada organización (ValbuenaGardeazábal, 2005).

La contabilidad puede entenderse como el enlace entre las actividades económicas de una empresa y quienes toman las decisiones (Nikolai \& Bazley, 2001).

Sistema de medición y comunicación de hechos económicos y sociales relacionados con un ente específico, que permite a sus usuarios juicios informados y decisiones conducentes a la colocación óptima de recursos y al cumplimiento de los objetivos de una organización (Aguiar, Cadavid, Cardona, Carvalho, Jiménez \& Upégui, 1998).

(...) La contabilidad entendida como disciplina científica al servicio del análisis económico básico (análisis de la circulación económica) y como herramienta auxiliar para la organización económica de las sociedades modernas (...) (García, 1997). La contabilidad pretende el conocimiento, interpretación y transmisión informativa de fenómenos circulatorios, generalmente económicos, compatibles con su metodología (Rodríguez-Ariza, 1992, Proyecto Docente para el Concurso al Cuerpo de Catedráticos de Universidad del Área de Economía Financiera y Contabilidad, Granada) (Gómez-López, s.f.). La contabilidad es una ciencia factual, social y económica, con un carácter esencialmente normativo.
Su objeto material es la realidad socioeconómica y su objeto formal el conocimiento cualitativo y cuantitativo de dicha realidad. Su fin es ofrecer información rigurosa, comprensible, imparcial, relevante acerca de esta realidad y de acuerdo con las necesidades específicas de todos aquellos que poseen un derecho razonable a la misma.

Para alcanzar dicho fin, utiliza una metodología que, en el marco del método científico, analiza, capta y procesa la realidad que constituye su objeto y posteriormente la comunica a sus destinatarios (Fernández Fernández-Cuesta, 1991).

De igual forma, el entendimiento de la riqueza se puede dar desde una concepción instrumental en la que se deba llevar fiel registro de esta pero la siguiente definición está pensada en la gestión de dicha riqueza, con un reconocimiento social y del entorno que la ubica tanto en el segundo como en el tercer enfoque:

'Ciencia que tiene por objeto la riqueza de las células sociales y que tiene la visión de un objeto específico pero observado dentro de un mundo global (Antônio) Lopes de Sá (1999, enero) Recursos naturais e empresa, en Jornal de Contabilidade, No. 262, Lisboa' (Niño-Galeano \& García-Fronti, 2003).

\section{Enfoque de reconocimiento del entorno}

Siguiendo la línea del apartado anterior, a continuación se ven algunas definiciones que ponen énfasis en el entorno con primacía en su 
carácter social y ponen la riqueza como un resultado de hechos tanto económicos como sociales.

La nueva contabilidad es hoy una disciplina que tiene por objeto el estudio y conocimiento del sistema de la circulación económica, empleando para ello como herramienta metodológica: el análisis circulatorio.

(...) La contabilidad, como cuerpo de conocimientos, es considerada una ciencia de carácter empírico; su naturaleza es económica y se ocupa del análisis económico (circulación económica, fenómeno de la riqueza...); tiene sus métodos propios de captación, medida y valoración, y su finalidad es tanto de tipo económico-financiero como social. La contabilidad, bajo su consideración como disciplina de naturaleza económica cuyo objeto de estudio es precisamente la realidad económica de los entes en todos sus niveles, debe reflejar cómo es la actividad de intercambio, y cómo se puede apreciar toda una circulación de la renta, la generación y distribución de una riqueza, a través de la utilización de los diferentes elementos propios de la disciplina, como son los métodos contables de medición y ordenación.

El contenido macro de la información contable debe buscar desarrollar una contabilidad socioeconómica, para superar las limitaciones presentadas por la contabilidad estrictamente presupuestal, patrimonial y/o financiera, permitiendo hacer énfasis en la medición de la productividad y rentabilidad social, como expresión que permite li- gar aspectos económicos y sociales, y como medidor de la gestión social de las unidades económicas que conforman una nación. Además, debe integrar y combinar aspectos contables con aspectos de naturaleza no contable, que reflejen con suficiencia aspectos cualitativos y cuantitativos de la gestión de cualquier organización y que permitan apreciar cuál ha sido el manejo de los recursos que las empresas han recibido de la sociedad. Este análisis ha de extenderse a la capacidad de determinar cuál ha sido su contribución al desarrollo económico y social de la comunidad o sociedad a la que pertenecen.

Los aspectos sociales involucrados en la contabilidad incluyen cuestiones tanto de índole micro como macro contable y, al no tener aún una identidad propia, las cuestiones sociales se solapan con las divisiones de la contabilidad más asentadas. Al intentar caracterizar las cuestiones sociales, destacamos que no solo se debe pensar en problemas económicos ni depende únicamente de la partida doble y de las cuestiones patrimoniales (Niño-Galeano \& García-Fronti, 2003).

La contabilidad, a más de ser fruto de la acción humana y de los hechos sociales, es uno de los elementos esenciales para motivar, impulsar y determinar nuevas acciones y nuevos hechos, particularmente los de producción y distribución de riqueza, entre otros. La teoría contable (ortodoxa) dice que la contabilidad es la base para la toma de decisiones, que tiene implicaciones para los diversos agentes. Así mismo, plantea que la 
información contable financiera debe poseer ciertas características que garanticen que la decisión sea la 'mejor', la 'correcta' (GómezVillegas, 2003).

Desde esta perspectiva y reconociendo los atributos de la contabilidad, esta se puede entender como un aspecto clave en el desarrollo que por supuesto no solo involucra aspectos financieros sino también sociales tal como lo menciona Jorge Tua-Pereda (2000).

De entre las posibles características actuales de nuestra disciplina, creo que hay que subrayar especialmente la relativa al impacto y la responsabilidad social de nuestra actividad... ... las razones de la íntima relación existente entre contabilidad y desarrollo son evidentes, si tenemos en cuenta que:

La primera es fuente primordial de información,

Que, a su vez, facilita la planificación macro y microeconómica

Promueve la creación y colocación eficiente de capitales,

Genera la confianza entre inversores y ahorradores,

Hace posible el correcto funcionamiento de las instituciones y unidades económicas, implica el desarrollo de los mercados de capitales.

$\mathrm{Y}$, en definitiva, constituye el motor de la actividad económica, fomentando el empleo racional de los recursos existentes en un país.

...Con todo ello, nuestra disciplina cobra una dimensión desbordante y se inserta en un marco de trascendencia suma: ser instrumento básico de optimización económica y social y, en consecuencia, contribuir al desarrollo económico...

En primer lugar, el profesional contable... Ha de ser consciente de que está inmerso en un sistema social donde priman unos valores y se desechan otros, y que su trabajo está condicionado por ellos. Así, si el experto contable calcula el beneficio es porque esta magnitud tiene una amplia repercusión social, de forma que marca decisivamente el éxito o fracaso de una actividad económica de quienes la llevan a cabo.

En segundo lugar, el experto contable ha de entender su actividad como una más de servicio a la colectividad. Esto implica que el producto informativo que obtiene después de aplicar sus técnicas e instrumentos de medida no es un fin, sino un medio que otros utilizarán para fines muy diversos. En tercer y último lugar, la relevancia social del experto contable o auditor viene marcada por el uso que se hace, externamente, de sus elaboraciones para dirimir intereses económicos. Cuestiones tales como el reparto del beneficio, las declaraciones fiscales, la intervención en disputas judiciales, la participación en expedientes de empleo etc., son algunos casos donde la actuación del experto contable puede ser decisiva.

Con lo anterior, es innegable que se puede reconocer dentro del núcleo del saber contable un carácter de responsabilidad social e incluso ético y moral; los siguientes apartados lo confirman: 
El proyecto de constituir a la contabilidad en una disciplina de naturaleza lógico-causal, como la ciencia natural clásica, no solo parece problemático sino quizás inconveniente... La pretensión positivista aun no encuentra nociones fundamentales que se consoliden como condiciones necesarias y suficientes que, de manera generalista y universalista, permitan explicar la diversidad y contingencia histórica de las herramientas y sistemas contables... ...Si la contabilidad es construida socialmente, muchas de sus técnicas y tecnologías, y por supuesto las teorías que soportan tal instrumental, tienen una carga moral significativa...

...en la medida en que 'Autoridad', 'Orden', 'Confianza', entre otros, son valores, que juzgamos como fines o propósitos loables y 'buenos', la contabilidad está atravesada por cuestiones y condicionantes morales...

Estos dos aspectos pueden connotar énfasis morales muy importantes, para que la contabilidad no solo aporte a la prosperidad económica privada y financiera, sino que trascienda hacia el desarrollo humano y social, la conservación de la diversidad y la consolidación de la democracia...

... El primer punto a resaltar es el enfoque contable hacia el tratamiento de las relaciones. Solo piénsese en tres nociones de la contabilidad:

La concepción de riqueza (y por tanto la idea de bienestar o de situación económica)

El concepto de valor (y los criterios de su tasación) y

La noción de utilidad (y los problemas de su definición en los marcos conceptuales)
En el capitalismo se considera por riqueza aquellos recursos que pueden reproducirse de forma ampliada como capital. Pero el capital no es el dinero, sino la relación de reproducción ampliada (Boltanski \& Chiapello, 2002). Así la riqueza no son las cosas, ni siquiera los recursos mismos...

...Por su parte, Amartya Sen (2004) y John Rawls (1997) han señalado, que la riqueza puede entenderse como una capacidad para servirse de los recursos y satisfacer necesidades y expectativas. A su vez, los recursos son objetos o servicios, que están cargados de sentido o de significación social. Por ello, se configura un proceso o ciclo que muestra la evolución de las cosas (elementos en el entorno; naturales o construidos por el hombre), tornándose en recursos (cosas con significación, sentido o uso social) y luego consolidándose como riqueza (capacidad de satisfacer necesidades y expectativas con los recursos disponibles).

En este sentido, poner en el centro de las relaciones para hablar de riqueza, implica realizar una de-codificación de lo que consideramos como activos, pasivos y del mismo patrimonio. Quizás, incluso, estas categorías no sean las más convenientes para nombrar las nuevas concepciones, pero, si la contabilidad debe dar cuenta de la riqueza, debería pues resignificar sus categorías básicas. Esta resignificación requiere entender el rol de las nociones vigentes (activo, pasivo, capital) en el orden moral vigente.

Desde las conceptualizaciones de valor y utilidad, las valoraciones contables no pueden verse como datos, simplemente expresados 
en soportes de transacciones o de datos del mercado, sino como evaluaciones sociales de lo colectivamente apreciado, deseable... en fin... de lo bueno.

El sistema no puede 'decir' que la utilidad es la 'apropiación privada de los recursos colectivos puestos en circulación en el proceso productivo'. Es decir, no se puede decir que la utilidad proviene del trabajo pagado, o de la apropiación de parte de las retribuciones equilibradas de otras. El orden moral implica que la propiedad de medios de producción como capital muerto, otorga derechos originarios de retribuciones superiores por los aportes.

Como segundo punto señalamos que la lógica de la dualidad bien podría aportar a dirigir la mirada contable hacia la comprensión de los procesos de circulación económica desde la termodinámica. Esta disciplina estudia la energía y sus procesos de transformación. Nuestro énfasis sería extender esta lógica de comprensión del equilibrio-desequilibrio energético a los procesos sociales.

La empresa genera utilidades porque desequilibra otro proceso en el sistema global. Comprender esto implica una ruptura moral fundamental, del que la contabilidad desde énfasis morales puede dar cuenta. Este énfasis daría inmenso potencial a la contabilidad para dar cuenta no solo de los procesos de enriquecimiento privado, sino de equilibrio social, bienestar global y desarrollo a escala humana (Gómez-Villegas, 2007).

Constituye un imperativo establecer nuevas dinámicas en la perspectiva de superar la tendencia convencional que reduce la con- tabilidad a tópicos estrictamente financieros generadores de lecturas parciales y usualmente descontextualizadas, desde donde el análisis se circunscribe precariamente a relaciones cuantitativas e insuficientes, privilegiando miradas de carácter profesionalizante (desarrollo de habilidades y destrezas) y reconociendo erráticamente a la contabilidad como técnica empírica de registro, sin consideraciones respecto a su condición y perspectiva disciplinar...

...Resulta entonces necesaria la ubicación de la contabilidad en el contexto de las ciencias sociales, con el propósito de superar la acepción convencional que da una gran centralidad al mundo de los registros sin mayores sustentos cognoscitivos y sin la observancia de algunas consideraciones acerca de la ciencia y del entorno económico-social y político-cultural.

La contabilidad en su construcción disciplinar, debe orientarse hacia la definición de una comunidad ideal de argumentación, una tradición escrita, una práctica científica y la precisión de puntos de encuentro o entronque con otras ciencias, como formas de participación en los procesos de transformación de las ideas y del conocimiento (QuijanoValencia, 2001).

Siguiendo con la relación de la contabilidad con otras ciencias, generalmente en definiciones de carácter tradicional hay una tendencia a vincularla con las ciencias económicas, jurídicas o matemáticas, relación que es indiscutible; no obstante bajo este enfoque de "Reconocimiento al entorno" algunos autores la vinculan 
con otras disciplinas, lo cual genera un análisis diferente.

Las múltiples relaciones con disciplinas como la gestión, la economía, las finanzas, la sociología, el derecho, entre otras, han tornado a la contabilidad en un saber altamente estratégico para el desarrollo y el bienestar económico y social. Las disciplinas de base científica se consolidan y avanzan, gracias a la investigación básica y aplicada (Consejo Técnico de la Contaduría Pública, CTCP). La contabilidad asume un papel de compromiso no solo frente a la realidad de las naciones del mundo, de sus grupos e individuos, sino frente a la humanidad. Su misión de reflejar la realidad financiera, económica y social es un servicio del cual se surten los individuos representantes de diversas profesiones (administradores, economistas, ingenieros, contadores, etc.), así como agentes sociales...

....La contabilidad como conocimiento o sistema disciplinal, abarca una serie de ramas que se ocupan de desarrollar el conocimiento desde diversas dimensiones, estas ramas son campos que pueden ser objeto del conocimiento (observación, medición, análisis, evaluación, control, etc.) contable. Con base en los planteamientos de (Richard) Mattessich, (Antonio) Calafell y (Leandro) Cañibano, puede señalarse la existencia de la contabilidad con unos supuestos básicos y unas ramificaciones que se desprenden de su expresión monetaria (unidades monetarias: pesos, dólares, euros, etc.) o no monetaria (unidades físicas: metros, kilos, horas, etc.) y de su carácter micro (a nivel de unidades económicas) o macro (a nivel de sistemas económicos)...

...El papel de la contabilidad frente a la realidad social ha sido el de construir modelos que simplifiquen la realidad social en variables de carácter humano, ecológico, ambiental, etc. Aún quedan interrogantes por resolver en relación con la equidad en la distribución de riqueza, la conservación de los recursos naturales y del ambiente, el reconocimiento y valoración de capitales alternativos, la responsabilidad social frente al uso de energías fósiles, valoración de alternativas energéticas (solar e hidrógeno), etc...

La contabilidad entonces debe ser concebida como una disciplina al servicio de la humanidad, como un conocimiento socialmente útil que tiene unos productos o herramientas (desarrollos teóricos, avances tecnológicos, técnicas, instrumentos y procedimientos) aplicables a la problemática contemporánea. La contabilidad social, unida (más no asumida) disciplinalmente a los desarrollos de la sociología, la psicología, la economía social, la ecología y la ingeniería ambiental, entre otras, podrá interpretar, conocer y mitigar las condiciones de miseria, atraso tecnológico, violencia, inequidad y corrupción que afectan a los ciudadanos, familias, pueblos o naciones del mundo entero.

De la forma como la contabilidad conciba la realidad social de los países y de cómo afronte la problemática adyacente, depende el nivel de contribución de los profesionales que la utilicen como herramienta de trabajo. La contabilidad social debe seguir avanzan- 
do y generando rupturas con el tradicional pensamiento contable y administrativo, para ello requiere del concurso de individuos de diversas profesiones que le brinden su apoyo intelectual.

Puede concluirse que la contabilidad ha venido construyendo, desde una óptica disciplinal, una serie de respuestas a las necesidades económicas y sociales. Desde un enfoque tecnológico e instrumental ha aplicado estas respuestas, a las necesidades concretas de los sistemas económicos y unidades económicas, a través de herramientas de medición, análisis y control. La contabilidad como campo de conocimiento, ha nacido, se ha desarrollado y pretende superar sus contradicciones en torno a las necesidades sociales; su razón de ser se centra en la satisfacción de las necesidades de las comunidades, del Estado, mercado y empresas, como expresiones de la naturaleza humana (Machado, 2003).

Para comprender la esencia de la contabilidad como conocimiento, necesitamos recurrir a disciplinas de variado origen; formales como las matemáticas y la lógica (que están exentas del proceso global) o de aplicación social como la economía, la administración, la sociología y el derecho (incididas por la globalización)

Los procesos decisionales de los usuarios y los tipos de información son analizados por la psicología, la lógica da soporte a la partida doble, términos como "patrimonio" tiene base en la antropología; las matemáticas se aplican en el diseño de modelos de proyección de la información contable; los sistemas de contabilidad de base legal y profesional dan origen al derecho contable; la economía suministra conceptualizaciones de la realidad que la contabilidad adopta como básicas; los enfoques de contabilidad social y medioambiental se basan en estudios de sociología y la ecología...

... No es que la contabilidad subsuma y se apropie de las hipótesis de aquellas otras disciplinas, pero las necesita para formular su método propio, a fin de lograr una mejor descripción y comprensión de su objeto (Gil, 2010).

Por último y como resultado de lo anterior, autores como Édgar Gracia-López (2010) hacen un llamado a la inclusión del criterio de "totalidad" que es la conclusión adecuada en cuanto al entendimiento de la empresa como parte de un "todo" en sus diferentes sentidos.

...Por lo general, la contabilidad se ha quedado con el análisis y contemplación de los fenómenos, con la simple descripción de los mismos, o con la versión instrumental de sumatoria de partes...

...El proceder explicativo de la contabilidad ha terminado apropiando solo una parte de la realidad (fragmentos) desarticulada de contexto globales - reales-y de fenómenos sociales, ambientales y culturales que emergen en las diversas formas de producción, apropiación y administración y consumo de la riqueza. Por esta vía idealista, el dato contable ha terminado despojado de su esencia, es decir, de sus cualidades intrínsecas que lo comunican con el mundo de la realidad. 
...Se descubre un criterio metodológico de 'totalidad' que se plantea como la simple suma de las partes, o en su mejor versión, como la interacción lógica de elementos que se postulan racionalmente, concepción que resulta contraria a otro criterio que asume la 'totalidad' en el sentido de la aprehensión de la esencia de la realidad como un todo estructurado... La aplicación del criterio de totalidad como la suma de las partes presenta limitaciones metodológicas, en tanto le atribuye al sistema posibilidades ilimitadas, desconociendo en la práctica la esencia de los fenómenos...

Dadas las nuevas condiciones de entorno, a la contabilidad le compete estructurar, informar, comunicar y controlar sobre los nuevos asuntos que se presentan en las organizaciones, tales como: lo referido a capital intelectual, innovación, ciencia y tecnología, productividad, sistema de relaciones, valores de distribución, comportamiento de objetivos, políticas y satisfacción de necesidades y consumos.

\section{Conclusiones}

A pesar de los cambios que se han generado con asuntos como la apertura económica y posteriormente la globalización, hay una corriente importante que sigue considerando a la contabilidad en su dimensión histórica e instrumental, que cuenta con un importante número de teóricos escribiendo entre 1990 y 2010 en este sentido y por ello sigue siendo uno de los enfoque más fuertes.

En contraposición, la corriente que reconoce su entorno y asume el carácter social de nuestra disciplina sigue siendo alterna e incluso ha sido catalogada por algunos como "débil". Numéricamente, los autores que se han dedicado a escribir en esta línea son menos.

Así como se pudiera catalogar de improbable lograr una definición única de contabilidad, debido a los diferentes criterios y aristas que se deberían tener en cuenta, verla en una sola dimensión también pudiera ser una forma de limitarla.

En una disciplina como la contable, las pyme (por considerarlas como el mayor número de organizaciones en países latinoamericanos) y en general, las empresas son las que deciden cuáles son el uso y el enfoque preponderantes, qué características queremos de la disciplina y a qué escenarios la vamos a llevar.

Una posible razón para considerar el enfoque social como débil es el bajo número de autores escribiendo en ella, pero otras razones pudieran ser el escaso número de profesionales que asumen en su ejercicio ese carácter o el exiguo número de empresas que contribuyen a la sociedad y reconocen su responsabilidad social desde todas sus dimensiones organizacionales.

Así, el escenario académico y el organizacional están llamados a continuar en un estudio juicioso sobre la disciplina y sobre su evolución de carácter holístico y que por ello tenga en cuenta todas sus dimensiones.

\section{Referencias}

Aarthur School of Business (s.f.). Accounting Dictionary. http://www.accountingdictionary.dk/regn/gbsp/regngbsp_index. php?site $=9 \&$ page $=0$ 
Agudelo Henao, L. F. (2006). Actividades multimediales para el desarrollo de competencias en contabilidad, una mirada desde los modelos pedagógicos constructivista y conceptual. Lúmina, Cuarto Foro Nacional de Educación Contable. Disponible en: http:// administracion.univalle.edu.co/Comunidad/ Memorias/evento1/archivos/Actividades\%20 multimediales\%20para\%20el\%20desarrollo\%20de\%20competencias\%20en\%20contabilidad\%20una\%20mirada\%20desde\%20 los\%20modelos\%20pedagogicos\%20constructivista\%20y\%20conceptual.pdf

Aguiar, Horacio; Cadavid, Luis Alberto; Cardona, John; Carvalho, Javier; Jiménez, Javier \& Upégui, María Eugenia (1998). Diccionario de términos contables para Colombia. Medellín: Editorial Universidad de Antioquia.

Arens, Alvin A.; Elder, Randal J. \& Beasley, Mark S. (2007). Auditoría: un enfoque integral. Aída Gabriela Valladares-Franyuti (trad.). México: Pearson Educación.

Biondi, Mario (1999). Teoría de la contabilidad. Buenos Aires: Ediciones Macchi.

Cañibano-Calvo, Leandro (1990). Contabilidad: análisis contable de la realidad económica. Madrid: Pirámide.

Carrillo de Rojas, Gladys (2004). Contabilidad para la gestión empresarial. Bogotá D.C.: Thomson.

Chaves, Osvaldo A.; Chyrikins, Héctor; Dealecsandris, Ricardo P.; Fronti de García, Luisa; Pahlen-Acuña, Ricardo J. M. \& Viegas, Juan Carlos (1998). Teoría contable. Buenos Aires: Ediciones Macchi.

Consejo Técnico de la Contaduría Pública, CTCP (s.f.). Consejo Técnico de la Contaduría
Pública. http://www.jccconta.gov.co/consejot/leercontenido.php?article_id=17

Contaduría General de la Nación, CGN (2009). Diccionario de términos de contabilidad pública. Bogotá: Contaduría General de la Nación, CGN. Disponible en: http://www. contaduria.gov.co

Delgado-González, Susana \& Ena-Ventura, Belén (2006). Contabilidad general y tesorería. Madrid: Thomson Editores.

Déniz-Mayor, José Juan; Bona-Sánchez, Carolina; Pérez-Alemán, Jerónimo \& SuárezFalcón, Heriberto (2008). Fundamentos de contabilidad financiera. Madrid: Delta Producciones Universitarias.

Escoto, Roxana (2004). El proceso contable. San José: Universidad Estatal a Distancia.

Fernández Fernández-Cuesta, M. P. (1991). Proyecto docente. Universidad de León.

Fierro-Martínez, Ángel María (2007). Introducción a la contabilidad. Bogotá: ECOE.

Fowler-Newton, Enrique (1992). Contabilidad básica. Buenos Aires: Ediciones Macchi.

García-García, Moisés (1997). Ensayos sobre teoría de la contabilidad (origen, desarrollo y contenido actual del análisis circulatorio). Madrid: Instituto de Contabilidad y Auditoría de Cuentas, ICAC.

Gil, Jorge Manuel (2010). Retos de la contabilidad frente a la globalización: perspectivas de la teoría y la investigación contable. En Conjunciones y disertaciones: Pensando la contabilidad en el siglo XXI, colección Perspectivas críticas de la contabilidad contemporánea, 1-19. Cali: Universidad del Valle.

Gómez-López, Roberto (s.f.). Enciclopedia y Biblioteca Virtual de las Ciencias Sociales, 
Económicas y Jurídicas. http://eumed.net/ cursecon/libreria/Contabilidad.pdf

Gómez-Villegas, Mauricio (2003). Contabilidad: comentarios sobre el discurso científico y los determinantes morales. Innovar, Revista de Ciencias Administrativas y Sociales, 22, 109-120. Disponible en: http://www.redalyc.org/pdf/818/81802212.pdf

Gómez-Villegas, Mauricio (2007). Pensamiento único y contabilidad: la posibilidad de la contabilidad como disciplina moral. Revista Facultad de Ciencias Económicas: Investigación y Reflexión, XV (2), 29-43. Disponible en: http://www.redalyc.org/articulo. oa?id=90915203

Gracia-López, Édgar (2010). Problemáticas de la teoría e investigación contables (algunos aspectos). En Conjunciones y disertaciones: pensando la contabilidad en el siglo XXI. Colección Perspectivas Críticas de la Contabilidad Contemporánea. Ciudad: Editorial.

Guajardo-Cantú, Gerardo (2004). Fundamentos de contabilidad. México: McGraw-Hill.

Gudiño-Dávila, Emma L. \& Coral-Delgado, Lucy del Carmen (2005). Contabilidad 2000. Bogotá D.C.: McGraw-Hill.

Horngren, Charles T.; Harrison, Walter T. \& Smith, Linda (2003). Contabilidad. Pilar Mascaró-Sacristán, Jorge Luis Blanco y Correa Magallanes \& Tomás Rodríguez-Couto (trads.), México: Pearson Prentice Hall.

Institute of Accounting Standards Board, IASB (2009). Normas Internacionales de Información Financiera (NIF). Disponible en: http:// www.nicniif.org/files/u3328/00_es_2009_ prelims_114.pdf
Instituto Mexicano de Contadores Públicos, IMCP (s.f.). Instituto Mexicano de Contadores Públicos. http://www.imcp.org.mx/spip. php?article18

Kieso, Donald E.; Kimmel, Paul D. \& Calvet-Pérez, Ricardo (2008). Principios de contabilidad. México D.F.: Limusa Wiley.

López-Ramírez, R. (2002). Introducción a las ciencias contables. Santiago de Cali: Unidad Central del Valle del Cauca.

Machado, Marco Antonio (marzo de 2003). Contabilidad y realidad social. Revista Asfacop, Asociación Colombiana de Facultades de Contaduría Pública, 6 (7), 107.

Martín-Amez, Fernando C. (2002). Diccionario de contabilidad y finanzas. Madrid: Grupo Cultural.

Meigs, Robert F.; Williams, Jan R.; Haka, Susan F. \& Bettner, Mark S. (2000). Contabilidad: la base para decisiones gerenciales. Bogotá: McGraw-Hill.

Nikolai, Loren A. \& Bazley, John D. (2001). Contabilidad intermedia. Adolfo Deras-Quiñones (trad.). México D.F.: Thomson Learning.

Niño-Galeano, Claudia Lucía \& García-Fronti, Inés Mercedes (2003). Algunas consideraciones para la reconstrucción del concepto de contabilidad para el presente siglo. Innovar, Revista de Ciencias Administrativas y Sociales, 21, 127-138. Disponible en: http:// www.redalyc.org/articulo.oa?id=81802109 Quijano-Valencia, Olver (6 de diciembre de 2001). Contexto, contabilidad e investigación: una lectura preliminar. Revista ASFACOP, Asociación Colombiana de Facultades de Contaduría Pública, 5 (5), 109-130. 
Rey-Pombo, José (2006). Contabilidad general. Madrid: Thomson Editores Spain.

Rivero-Romero, José (1991). Contabilidad financiera. Madrid: Centrum.

Samuelson, Paul Anthony; Nordhaus, William D. \& Mandel, Michael J. (1996). Economía. McGraw-Hill.

Sánchez-Cárdenas, David Eduardo (2003). La contabilidad a mi alcance. Bogotá, D.C.

Sánchez-Fernández de Valderrama, José Luis (2008). Teoría y práctica de la contabilidad. Madrid: Ediciones Pirámide.

Sinisterra-Valencia, Gonzalo; Polanco-Izquierdo, Luis Enrique \& Henao, Harvey (2001). Contabilidad: sistema de información para las organizaciones. Emma Ariza Herrera (ed.). Bogotá, D.C.: McGraw-Hill.

Sinisterra-Valencia, Gonzalo; Polanco-Izquierdo, Luis Enrique \& Henao, Harvey (2005). Contabilidad: sistema de información para las organizaciones. Bogotá: McGraw-Hill.

Sunder, Shyam (2007). Teoría de la contabilidad y el control. Bogotá: Universidad Nacional de Colombia.

Tascón-Fernández, María Teresa (1995). La contabilidad como disciplina científica. Contaduría, Universidad de Antioquia, 26-27, 65-111.
Tua-Pereda, Jorge (2000). En torno a la docencia de la contabilidad una reflexión personal. Porik An, Revista Facultad de Ciencias Contables, Económicas y Administrativas, 3-4.

Valbuena-Gardeazábal, José Ignacio (2005). La organización empresarial y la contabilidad. @puntes Cont@bles, Revista de Contaduría Pública, 7, 33-54. Disponible en: http://revistas.uexternado.edu.co/index.php?journal $=$ contad $\&$ page $=$ article\&op $=$ view\&path $\% 5$ B\%5D=1296\&path\%5B\%5D=1233

Wilkinson, Joseph W.; Cerullo, Michael J.; Raval, Vasant \& Wong-On-Wing, Bernard (2000). Accounting Information Systems. Essential Concepts and Applications. New York: John Wiley \& Sons, Inc.

- Fecha de recepción: 30 de agosto de 2013

- Fecha de aceptación: 17 de enero de 2014

\section{Para citar este artículo}

Uribe-Bohórquez, María Victoria (2014). Caracterización de la evolución en el concepto de contabilidad de 1990 a 2010. Cuadernos de Contabilidad, 15 (37), 239259. 
